\title{
LA CALIDAD COLABORATIVA: UN NUEVO CONCEPTO PARA LA CALIDAD TURÍSTICA
}

\author{
Marta Candau del Cid
}

Cimac. Consultoría de Marketing y Sistemas de Gestión de la Calidad

\section{RESUMEN}

La tecnología 2.0 ha transformando a los consumidores en fuentes de creación y retroalimentación para las empresas, situándolos como elementos clave para la gestión de la calidad. El presente artículo analiza los sistemas de gestión de calidad (SGC) turística española: la marca Q de calidad y el distintivo SICTED, en las web y las redes sociales (Facebook y Twiter) en la industria hotelera de Andalucía. La investigación demuestra que los actuales SGC turística han obviado los factores que generan valor en los nuevos consumidores: Posicionamiento, visibilidad, comunicación e interactuación. Sus procesos no implementan las estrategias de marketing asociadas a la tecnología 2.0, para potenciar sus marcas como referente de confianza. Han eludido a los nuevos consumidores, piezas esenciales de las nuevas estructuras empresariales. Esta debilidad ha sido utilizada por los "sellos influencers", para desplazarlos como referentes de confianza. Los SGC turística deben transitar de herramientas de correcta gestión empresarial a garantes de una mejora continua; visible, comunicada y retroalimentada por los nuevos consumidores. Adaptándose a la nueva etapa de la gestión de calidad, la Calidad Colaborativa.

Palabras clave: Calidad turística; Marketing Turístico; Fidelización de cliente; Imagen de marca.

\section{Collaborative quality: a new concept for tourism quality}

\section{ABSTRACT}

The technology 2.0, has transformed consumers into informed individuals, content generators and sources of feedback for companies, positioning themselves as key elements to managing quality. This article analyses the positioning, visualization, communication

Fecha de recepción: 1 de junio de 2018.

Fecha de aceptación: 4 de octubre de 2018.

Cimac. Consultoría de Marketing y Sistemas de Gestión de la Calidad. C/ Canalejas, 14. SEVILLA (España). E-mail: mcc@cimac.es 
and interaction that current Spanish tourism systems for quality management: the Q mark for quality and SICTED [Complete System for Tourism Quality in Spanish Destinations] emblem, on websites and social media (Facebook \& Twitter) in the Andalusian hotel industry. This investigation shows that current tourism QMSs have ignored factors that generate value for new consumers: positioning, visibility, communication and interaction. Their processes do not include marketing strategies associated with technology 2.0 , to promote their brands as references of trust. They have removed consumers from the equation, essential pieces in any new business structure. This weakness has been utilized by "Influencer marks" in order to displace QMSs as references of trust. Tourism QMSs should transition from tools of correct business management to guarantors of continual improvement that is visible, communicated, receives feedback to new consumers. QMSs must adapt to the new era of quality management, the Collaborative Quality.

Keywords: Tourism quality; Tourism Marketing; Customer loyalty; Brand identity.

\section{INTRODUCCIÓN}

A lo largo de la historia, las transacciones comerciales se han regulado mediante la norma básica de la oferta y la demanda. Esta relación ha sido inalterable, aunque ha variado la forma de satisfacer al mercado. En la era industrial donde la oferta era inferior a la demanda, los servicios y productos se diseñaban para atender las necesidades de un mercado de masas, donde el objetivo se centraba en la estandarización. Se aplicaron las economías de escala y se redujeron los costes, logrando así que los servicios y productos fuesen accesibles en precio y llegaran al mayor número de consumidores. La evolución tecnológica del siglo XX y XXI ha provocado cambios en los consumidores (demanda) y en el mercado de los servicios y productos (oferta). Estos últimos se han modificado para satisfacer las necesidades de los consumidores, que solicitan productos específicos que se ciñen a sus gustos e inquietudes socio-culturales (Palafox, 2014). Esta evolución y especialización, unida al desarrollo de los sistemas de producción, ha fragmentado el mercado de consumo en múltiples productos y servicios, "hemos creado un mundo en el que la mayoría de los productos son invisibles" (Godin, 2008:16).

Los servicios y productos que quieran diferenciarse y, por tanto, tener éxito en el actual mercado saturado por consumidores sin tiempo (Vieites y Barros, 2011) y con una capacidad de asombro limitada deben tender a ser como "la vaca púrpura", productos extraordinarios que interesan realmente a la gente (Godin, 2008; Esteban, 2014).

La nueva era tecnológica y de la comunicación interactiva ha revolucionado el consumo. El cliente ha modificado su manera de relacionarse y sus hábitos de compra. Su cambio de la actitud y aptitud en las transacciones comerciales, unida a la nueva estructuración de las empresas producidas por las TICs, y a los avances del transporte han dado lugar a organizaciones empresariales desconocidas hasta la fecha (Palafox, 2014). Esta transformación es lo que algunos autores han llamado la cuarta revolución industrial (López \& Castillo, 2016; Schwab, 2016). 
La cuarta revolución comienza en el año 2004, cuando se acuña el término "web 2.0", creado en la sesión de "brain stroming" entre Tim O’Reilly y MeiaLive (Kaplan y Haenlein, 2010). Esta revolución es considerada la era de la participación debido a la co-creación de contenido, el emitido por las empresas y el producido por los consumidores (Deza, 2007). En ella, los individuos crean ideas y entretenimiento, además de consumirlos. Con esta tecnología se pasa de consumidores a prosumidores (productor + consumidor), es decir, "han pasado de ser individuos aislados a conectados. Cuando toman decisiones, ya no lo hacen desde el desconocimiento, sino que están informados. Ya no son pasivos sino una fuente activa de retroalimentación de información para las empresas" (Kotler et al., 2011: 27).

En esta misma línea, existen semejanzas entre la evolución del consumo y la transformación que ha desarrollado el marketing para amoldarse al mercado en cada etapa, definiendo estrategias que posicionan los productos de forma preferente (Kotler et al, 2011; Velilla, 2010). La imagen y el posicionamiento de marca se han convertido en las estrategias centrales de la relación entre las organizaciones y sus clientes, y en la protagonista de un diálogo que incrementa el valor percibido (Domingo, 2013). Las empresas, conscientes del impacto que las redes sociales tienen en la imagen, las han utilizado como herramientas de marketing. Su bajo coste, inmediatez, capacidad de difusión y gran implantación en el mercado está permitiendo que sea empleada para la promoción de productos y servicios, la comunicación con clientes, y más importante aún, para el conocimiento del comportamiento y preferencias del consumidor, además de como canal de ventas (Harris y Rae, 2009).

En los últimos 60 años, el marketing ha pasado de una perspectiva centrada en el producto (marketing 1.0), a una basada en el consumidor (marketing 2.0) para aterrizar a principios de este siglo XXI en una visión centrada en el ser humano y en la que la rentabilidad se concilia con la responsabilidad corporativa (marketing 3.0) (Erragcha y Romdhane, 2014; Kotler et al., 2011; Vieites y Barros, 2011). Si se compara esta evolución del marketing con las etapas de la calidad (Anexo I) se percibe el paralelismo de las dos primeras etapas y se vislumbra el nacimiento de la tercera en el horizonte. En la etapa 1.0, la calidad estaba centrada en la mejora y estandarización, el proceso de calidad 2.0 se centró en la normalización, sectorización, regularización y certificación de los procesos. Actualmente, la calidad se enfrenta al nacimiento 3.0 basada en la transmisión de valores morales, credibilidad y confianza al consumidor, a través de sus sellos de certificación, a la que denominaremos calidad colaborativa.

Este cambio afecta a la forma de entender y gestionar la calidad, donde la opinión de los viajeros tiene un papel preponderante en la valoración de los servicios, a través de los medios sociales (González et al., 2014). La calidad colaborativa incorpora este enfoque además de que incluir y actualizar los objetivos y conceptos previamente establecidos (Anexo II). Esta fase requiere de la colaboración de los usuarios, de forma activa, para convertirla en herramienta de valor hacia el empresario y el cliente. Una colaboración que vertebrada por estrategias de marketing pondera la calidad, que ya no sólo se produce y gestiona, sino que también se hace visible y se comparte como parte fundamental del servicio que completa a la imagen (Candau, 2016). 
La etapa de la calidad colaborativa es la consecuencia evolutiva de los cambios tecnológicos acaecidos en el mercado. En esta etapa, los SGC precisan de la colaboración e interacción activa con los stakeholders de la organización, al objeto de convertirse en herramientas generadoras de valor para el empresario. Deben promover un flujo de información, generando una creatividad masiva, que trabajada éticamente y con inmediatez, garantice productos y servicios que mejoran continuamente, conquistando las necesidades de la nueva era de clientes; a los que hay que satisfacer tanto de forma funcional, emocional como espiritual. Las relaciones deben ser establecidas de forma transparente y vertebradas por estrategias de marketing. En esta etapa, la calidad no sólo debe ser producida y gestionada, sino que también debe ser interactiva, visible, comunicada y vendida al cliente. Convirtiéndose en garantía real para los clientes. (Candau, 2016).

A modo de ejemplo, las compañías aéreas han adaptado sus procedimientos a las TICs creando una metodología que aporta inmediatez y cercanía en la resolución de las quejas, y facilita la incorporación de sugerencias. La calidad colaborativa se asienta tanto en la empresa como en sus stakeholders, promoviendo la innovación colaborativa a través del acercamiento, diálogo y participación. Las características que definen la calidad colaborativa son la innovación a través de la creatividad masiva, la colaboración bajo un código ético y la comunicación directa e inmediata entre los miembros de la red. Estas premisas garantizan trabajar con honestidad y transparencia, poniendo el conocimiento al alcance de todos (Carrasco y Lorenzo, 2015).

El objetivo de la calidad colaborativa se centra en satisfacer al cliente de forma funcional, emocional y espiritual (Candau, 2016), puesto que una de las consecuencias de la globalización ha sido la generación de una economía interconectada, que no es sinónimo de equitativa. Esta paradoja ha llevado a cambiar el perfil del consumidor, que ha pasado a ser más consciente y sensible, no sólo a su entorno cercano sino también en el global (Inglada y Sastre, 2014).

La prestación del servicio turístico de calidad depende de factores estratégicos, territoriales, medioambientales y patrimoniales muy diversos. Una de las mayores dificultades para el empresario turístico reside en la naturaleza transversal del turismo que engloba competencias territoriales diversas y ordenamientos jurídicos dispares que deberán ser armonizados con el fin de infundir seguridad al empresario (Fernández, 2013) de forma que puedan ofrecer a sus clientes el bienestar que busca en el entorno que visita.

Las empresas interesadas en satisfacer las necesidades de sus consumidores y usuarios han ubicado a sus clientes como punto estratégico. De este modo, estas empresas impregnan valores a su marca (Costa, 2014), y aportan diferenciación ante los ojos de los consumidores. Éstos buscan experiencias y modelos de negocio que les desarrollen su faceta espiritual, centrado en los valores (Novo, 2014). Éste es el nuevo escenario en el que compiten las empresas (Kotler et al., 2011). Los certificados de calidad se posicionan como referentes generadores de confianza. Sus logotipos identifican a las empresas que ofrecen servicios acordes con sus necesidades primarias y sus anhelos más altruistas como la preservación del medioambiente, el respeto a los derechos humanos, la responsabilidad social, la seguridad en el comercio electrónico, etc. (González et al., 2014; Viñarás et al., 2015). Este cambio debe ser adecuado, adaptando los proce- 
dimientos a las TICs, generando una herramienta capaz de proporcionar inmediatez a los empresarios con los stakeholders (Casanueva et al., 2013, Cravero y Alza, 2012).

Las tecnologías han establecido diversos comportamientos que están generando nuevas normas sociales (Schwartz, 2009). Las empresas han reconocido la necesidad de ampliar su marca y servicios en el canal online, para ofrecer más posibilidades y más experiencias (Deza, 2007). La evolución de los medios se dirige hacia lo digital, lo que permite la interacción con el consumidor de forma continua (Simmons, 2007). En cambio, la comunicación unidireccional ya no funciona. Las marcas que se mantienen en ello fracasan en el mundo digital (Cuesta y Alonso, 2010). Con la llegada de las redes sociales, se evidencian nuevos espacios que permiten al público opinar y compartir, además de comunicarse, como se evidencia en plataformas como Twitter, Facebook o YouTube (González y Contreras 2012). Con estas herramientas de participación, gestionar el conocimiento debe ser el objetivo de cualquier organización (Domingo, 2013; Ricelly et al., 2017).

El presente artículo examina el posicionamiento, la visibilidad, la comunicación e interacción que mantienen los hoteles andaluces certificados con los Sistemas de Gestión de Calidad (SGC) Turísticas; SCTE-Sectores con la marca Q de calidad y SCTE-Destinos con el distintivo SICTED con sus clientes en las páginas webs y redes sociales (Facebook y Twiter). Así mismo se analiza la implementación de las tecnologías de la información y de la comunicación (TICs) para establecer redes de colaboración con sus stakeholders al objeto de promover la mejora continua de sus SGC. De esta forma, se reconoce si los SGC turística son valorados por los empresarios como elementos distintivos en el mercado y si su implementación ha contribuido la adecuación de la revolución tecnológica. Esto permitirá verificar si los distintivos Q y SICTED son considerados por los usuarios como elementos decisivos en la compra ó si por el contrario, estos sistemas han quedado desfasados y deben amoldar sus sistemas a la etapa de la calidad colaborativa.

\subsection{Los sistemas de Gestión de Calidad Turística: SCTE-Sectores con la marca Q de calidad y SCTE-Destinos con el distintivo SICTED}

Los gestión de la calidad es elemento fundamental para competir en el mercado de servicios. Sin la implantación de un sistema de gestión de calidad, sea certificado o no, se pierde la homogoneidad del servicio, descompensandose el binomio calidad/precio adquirido por el cliente en el momento de la compra. El incumplimiento de este binomio provoca la insatisfacción del cliente que ve truncadas sus espectetivas derivandose en reclamaciones al proveedor de servicios turísticos (Candau, 2016).

España fue el primer país en crear el SGC turística, a principios de los noventa con el objetivo de reposicionar la imagen del turismo español en el mercado global, tras la crisis turística de finales de los 80. Bajo el paraguas del Plan Integral de la Calidad del Turismo Español, en el periodo de 2000 a 2006, comienza a implantarse el enfoque del aseguramiento de la calidad con la creación del SCTE (Sistema de Calidad Turística Española). El proyecto nació con dos objetivos principales: El primero, dotar a las empresas de una metodología y un sistema de gestión la mejora continua de la calidad. El segurndo, respaldar institucionalmente la imagen de marca de España como destino de calidad turística (Camisón, 2004; García-Henche, 2005; Calidad Turística, 2016). 
La marca Q calidad y el distintivo SICTED (Sistema Integral de Calidad en Destino) son los SGC turística utilizados que utiliza el SCTE. Comienzan a desarrollarse en el 2000, aunque la Q tiene su origen en 1996 bajo el amparo del ICHE (Instituto Calidad Hotelera Española). Basados en normativas que exigen estándares de servicio, gestión y mantenimiento, ambos sistemas tienen como finalidad aportar a los establecimientos turísticos y entidades que las ostentan: prestigio y diferenciación en el mercado (Huertas-García, 2005). El SGC Q está destinado a los agentes turísticos y es regulado por el ICTE. Mientras que el SICTED tiene como objetivo el Destino turístico y está desarrollado y regulado directamente por la SET (Secretaria de Estado para el Turismo). Ambos SGC son certificados y evaluados en auditorias, considerando la satisfacción del cliente la mejora continua como bases de su desarrollo. (González et al., 2014; Álvarez et al., 2017).

\subsection{Posicionamiento y Visualización de logotipos de los SGC en webs y redes sociales}

Uno de los grandes desafíos que afrontan las empresas es hacer frente a la elevada sensación de desconfianza por parte de los usuarios que operan en internet. Para actuar frente a tal hándicap se han ideado los certificados de calidad. Tales instrumentos constituyen sugerentes logotipos que se sitúan en los sitios web de las empresas comprometidas activamente con los mismos. Su visión, por parte de los potenciales clientes, reduce considerablemente la sensación de desconfianza frente a la empresa en cuestión (López et al., 2013; López y Monroy, 2013).

La incorporación de los logotipos de calidad a las páginas webs y redes sociales permiten a los consumidores optar entre aquellos públicamente comprometidos con las mejores prácticas empresariales y los que carecen de semejante compromiso (De Bruin et al., 2005). Los primeros ofrecen un valor añadido al servicio comercializado. La presencia de una etiqueta de confianza supone que el empresario que la ostenta asuma y se comprometa a cumplir el código de conducta y buenas prácticas del mismo (López y Redchuk, 2015). Constituyen un reconocimiento de la calidad de las empresas que lo hayan obtenido y con la exhibición de los distintivos buscan mejorar su posicionamiento en el mercado, lo que se traduce en una ventaja respecto a la competencia y se beneficia del prestigio del logotipo (López y Castillo, 2016).

Atraer la atención del usuario hacia los logotipos de calidad depende de la disposición ó posición que éstos tengan en sus páginas webs y redes sociales. La posición dentro de la "world wide" varía. Cuando el logo figura en el título es más relevante, que si aparece en el cuerpo (Codina y Marcos, 2005). El impacto de visualización es mayor si los logotipos quedan ubicados en la parte superior de las páginas webs, sin que tenga que desplazarse por la misma. Los puntos básicos de un diseño web convincente, es la zona "above the fold" que recibe aproximadamente el $80 \%$ de la atención de los usuarios (Laja, 2013). Este término anglosajón proviene de la imprenta de dicho país, dónde los periódicos se distribuyen doblados por la mitad. De esta forma, los usuarios lo primero que perciben son los títulos de la portada que están por encima de la zona por la que se dobla el periódico, denominada fold. Esta sección, por tanto, es el sitio 
óptimo de visualización. Si se desea atraer la atención del usuario, ésta es la ubicación donde se debe aparecer lo más destacado. El espacio que no está comprendido dentro de la franja descrita se designa como "below the fold" que traducido literalmente significa "debajo del pliegue".

Esta teoría se ilustra en el dicho popular "quien no es visto, no es recordado". "Ello es imperativo categórico del mercado. En los mercados turísticos no estar en red significa un anacronismo productivo" (Ricelly et al., 2017: 635).

\subsection{Comunicación y difusión de la certificación de los SGC}

La comunicación busca la movilización del cliente potencial. Las etapas que marcan el grado de vinculación que el consumidor adquiere tras someterse a la comunicación de la marca son: 1) la comunicación genera conocimiento de la marca; 2) consigue que el consumidor perciba sus valores y beneficios; 3) genera la intención de compra; 4) persigue que el consumidor sea fiel a la marca (Chaves, 2006). La no comunicación de los valores de la certificación de calidad elimina el valor añadido que puede dar a la marca turística a la que complementa.

"Un logotipo de calidad podrá ser realmente eficaz cuando sea ampliamente conocido por el público al que se dirige" (López y Castillo, 2016:79), Estos autores analizan los certificados de calidad del mercado electrónico europeo enumerando los criterios que una marca de confianza debe transmitir en las webs para garantizar credibilidad tanto en sus relaciones B2C como B2B. Algunos criterios basados en la claridad y la accesibilidad de la información, que destacan como puntos más relevantes, son:

- La publicación de información sobre los certificados de calidad que se ostentan.

- La difusión de los requisitos que son necesarios para ser reconocido.

- Los integrantes del sistema del certificado.

En este mismo sentido, los sistemas de marcas de confianza ó certificados de calidad deberían publicar un informe anual bajo un lenguaje claro, entendible, conciso y de fácil acceso al cliente (De Bruin et al., 2005). Este argumento explica la importancia de comunicar al público el significado de las marcas de confianza y, por tanto, la importancia de la difusión de la certificación Q y SICTED tanto en las páginas webs como en las redes sociales de las empresas certificadas.

Las tecnologías de la información y de la comunicación (TICs) están creando nuevas formas de relacionarse entre las empresas y los consumidores, que hubiesen sido impensables en el siglo XX. En este sentido, destaca el enorme impulso que han tomado las redes sociales y los nuevos medios de comunicación (Cuesta y Alonso 2010). Esta situación exige de las marcas y las empresas que se comuniquen como entes sociales en estos medios (Domingo, 2013; González y Contreras, 2012).

Al igual que en su día Lucio Anneo Séneca dijo "si me ofreciesen la sabiduría con la condición de guardarla para mí sin comunicarla a nadie, no la querría". Esta afirmación hace reflexionar sobre el sentido que tiene adherirse a un SGC turística, que luego no es comunicado a los clientes. 


\subsection{El poder de los "sellos influencers" frente a los certificados de calidad}

A principios de siglo XXI, las marcas estaban perdiendo su capacidad de diferenciación y se veían amenazadas por los grandes grupos de distribución. Se habían perdido los planteamientos clásicos de comunicación y comenzaba un nuevo modelo (Ollé y Riu, 2009). La tecnología 2.0 había revolucionado estructuralmente la economía, siendo el centro de las nuevas formas de comunicación. Esta revolución se convirtió en un tsunami en el ámbito del marketing, donde el nacimiento, difusión e influencia de las redes sociales ha provocado que la comunicación esté cada vez más fragmentada y bajo el control de los usuarios, en lugar de la marca (Aaker, 2010). La figura del prescriptor ha sido uno de los recursos más utilizados en la comercialización de servicios y productos. La democratización de la influencia de la comunicación ha generado que el poder de recomendación se haya extendido prácticamente a cualquier individuo que sea activo en las redes sociales (Hennig-Thurau et al., 2010).

La recomendación que realiza alguien que se conoce ó está dentro del grupo referencial tiene más influencia sobre un individuo que cualquier acción de marketing. La eclosión de las redes sociales y la posibilidad que los entornos 2.0 han ofrecido a la hora de compartir sus opiniones, ha provocado que dicha prescripción, también denominada "boca-oreja", se haya incrementado (Cantallops y Salvi, 2014; Castello y del Pino, 2015; Hernández-Méndez et al,.2015). Conscientes de este poder, las agencias on-line han extrapolado este recurso a sus plataformas digitales turísticas creando los premios a la calidad dirigidos a un establecimiento y otorgados por los propios clientes. De esta forma, aprovechan el poder de prescripción y recomendación que tienen los testimonios. Algunos autores refuerzan la importancia de la recomendación cuando indica "un cliente fiel no es sólo aquel que repite transacciones, sino el que por encima de todo, se siente satisfecho y orgulloso, y así se lo cuenta a los demás" (Celaya, 2008: 227). Los testimonios en la red generan contenido orgánico o no pagado sobre la marca y la posicionan en los metabuscadores, mejorando su visualización en los clientes potenciales. (Subrami y Rajagopalan, 2003).

Los "sellos influencers" son un producto del marketing creados por metabuscadores del sector turístico, con el objetivo de atraer la atención y ganarse la confianza de los usuarios. Bajo un logotipo de calidad utilizan la tecnología 2.0 animando a los usuarios a describir sus experiencias y puntuar los servicios de los establecimientos hoteleros utilizados, promoviendo la recomendación C2C. Existen dos tipos estándar de sellos influencers. Por un lado, los prescriptores, que son recomendaciones donde se indica la puntuación de los usuarios. Y por otro, los instaurados como premios a la excelencia por ser los mejores puntuados en una zona geográfica determinada. En ambos se da la casuística de la prescripción C2C. (Candau, 2016)

El fin de los SGC es proporcionar la satisfacción total y, por tanto, la diferenciación con respecto a la competencia en el mercado. Los certificados de calidad otorgados por las agencias o plataformas turísticas han sido creados para despertar y captar la confianza de los usuarios, basándose en la experiencia y lealtad que han desarrollado otros usuarios por estas empresas. Estas bondades vinculadas con el marketing relacional no 
están incluidas en los sistemas de calidad Q y SICTED, por lo que es interesante investigar la relevancia que están obteniendo los sellos de calidad influencers (TripAdvisor, Trivago, Holiday Ckeck, etc.) frente a los sistemas Q y SICTED.

\subsection{La interacción con los clientes en la era digital}

La web 2.0 ha supuesto un cambio en el panorama turístico y, más concretamente, en el hotelero. Los clientes acuden a las webs y redes sociales para ampliar la información sobre el destino fijado antes de realizar su reserva. Facebook, Twiter ó metabuscadores como Tripadvisor son ejemplos de ello. (Borràs et al., 2014)

A través de estos medios, los usuarios se retroalimentan de imágenes, opiniones y experiencias del establecimiento. Asimismo, las empresas hoteleras tienen acceso a las opiniones de la estancia de sus clientes, así como a los precios, servicios y productos ofertados por su competencia (Diana-Jens y Ruibal, 2015). "Las empresas ya no tienen el control absoluto de sus marcas, ahora compiten con el poder colectivo de los consumidores (...) y tienen que colaborar con ellos. Esta colaboración comienza cuando (...) se escuchan a los consumidores para entenderles y conocer mejor el mercado (...). Los mismos pasan a desempeñar un papel fundamental en la generación de valor a través de la creación conjunta de productos y servicios" (Kotler et al., 2011: 26). El cliente es conocedor que en sus manos está parte de la reputación de una marca (Del Pino et al., 2013: 184) (Crick y Spencer, 2011).

Aunque las empresas han sumado el potencial de las redes sociales implementándolas en sus estrategias de marketing con el objetivo de crear y mantener vínculos con los huéspedes: antes, durante y después de la estancia (Veledo, 2016), necesitan de una mayor exposición a internet y adoptar mejores prácticas en sus estrategias digitales de comercialización (Parra y Martínez, 2014). Los hoteleros se enfrentan a la decisión de innovar e invertir en el uso de las TICs, aspectos que serán críticos para su posicionamiento y competitividad (Murphy et al., 2006). Por ello, el uso eficiente de redes sociales resulta efectivo para la captación y fidelización de clientes (Mellinas et al., 2016).

La generación de confianza en los clientes y la interación que la empresa tiene con los mismos, es decir, el Business to Consumer (B2C) ha suscitado gran interés en el mundo académico (Brodie et al., 2011; Lai, 2014), debido a su vinculación con los indicadores de desempeño de marca como el crecimiento de ventas, la participación de los clientes en el desarrollo de productos o la retroalimentación de los clientes (Van Doorn et al., 2010). En la industria del turismo, se ha comprobado que la interacción con los clientes fomenta la lealtad y afianza la confianza en la marca (Harrigan et al., 2017). La interacción con los clientes es facilitada por las redes sociales y las tecnologías aplicadas en web.

Mediante la investigación de la interacción que mantienen los hoteles certificados con sus clientes se evidencia el nivel de implantación de aplicaciones 2.0. Haciendo posible la construcción de una participación con los clientes que promueva y genere la mejora continua en la calidad del servicio. Atrayendo la confianza del cliente, al atender con rapidez sus demandas, ofertar servicios que hayan sido demandandado y construyendo de forma conjunta (empresa-clientes) servicios que satisfaga al 100\% las espectativas de los clientes. Se genera la transparencia que origina la lealtad, que redundará en el crecimiento de las las ventas. 
La interacción con los clientes es una realidad que debe ser implementada por aquellas empresas que deseen ofrecer a sus clientes una mejora continua de sus servicios y productos. Los resultados del nivel de interacción dentro de las empresas certificadas por Q y SICTED con sus clientes, medirán la adaptación de sus respectivos SGC a la tecnología digital, así como si la mejora continua en estas empresas ofrece a sus clientes como parte esencial de su servicio.

\section{METODOLOGÍA}

La revolución 2.0 ha incidido de forma impactante en el sector del turismo cambiando la relación que los clientes mantienen con las empresas. El presente estudio es fruto de una reflexión sobre los cambios que debe adoptar la calidad turística con objeto de amoldarse a las innovaciones tecnológicas y de interactuar en esta cuarta revolución industrial.

Para alcanzar dicho objetivo el trabajo analizar los resultados de la exposición y difusión que tienen los SGC turística; Q de calidad y SICTED en internet. El análisis de los sitios web y redes sociales (Facebook y Twiter) de los hoteles andaluces certificados con ambos distintivos darán respuesta a los siguientes ítems;

- Valoran los empresarios estas certificaciones como diferenciadores destacables para la comercialización de sus establecimientos.

- La implementación de estos sistemas ha contribuido a la adecuación de la gestión hotelera a la tecnología 2.0.

- Son estos las certificaciones Q y SICTED considerados por los usuarios como referentes decisivos en su elección de compra.

Estos ítems determinaran si los actuales SGC turística están adecuados a los nuevos tiempos ó han quedado desfasados siendo obligados a revisar su modelo adecuándolo a la calidad colaborativa.

Los parámetros utilizados para realizar el estudio, no se han centrado exclusivamente en la usabilidad y el posicionamiento pues resulta insuficiente un análisis global (Fernández-Cavia y Huertas-Roig, 2009; Fernández-Cavia et al., 2013; Luna-Nevarez y Hyman, 2012) ampliándose a:

- Aspectos técnicos (posicionamiento y visibilidad).

- Aspectos informativos (comunicación).

- Aspectos relacionales (relevancia social media e interacción).

Siguiendo esta meta, las hipótesis planteadas son:

H1: ¿Están bien posicionados y son visibles los logotipos de calidad (Q de calidad y SICTED) en las páginas webs y en los perfiles de las redes sociales de los hoteles andaluces?

H2: ¿Comunican los hoteles certificados a los clientes los beneficios que les ofrece el que sus servicios estén certificados? 
H3: ¿Qué relevancia tienen los sellos de calidad “influencers” frente a los certificados de calidad?

H4: ¿Los hoteles han amoldado su gestión de calidad e interacción con los clientes a la era digital?

Para ello, se evalúan 33 indicadores (Anexo III), donde se comprueba la presencia del elemento de manera dicotómica. Este cuestionario revela el grado de interacción que tienen las empresas hoteleras con sus clientes y su adaptación a la era digital. La aplicación de este sistema de evaluación en internet se llevó a cabo entre junio 2015 y noviembre de 2016, un periodo de tiempo prudente con el fin de minimizar el riesgo de encontrar cambios importantes.

Se eligió estudiar las empresas hoteleras ya que al ser éstas las primeras en adecuarse al canal de comercialización de las nuevas tecnologías (Schegg et al.,2008; Cetinkaya, 2010), se admitió que se debían ser las empresas turísticas más avanzadas en la adaptación a los avances tecnológicos.

El alcance del estudio abarca el total de los establecimientos hoteleros que han implantado los dos SGC: el certificado de la Q de calidad y la distinción de SICTED en Andalucía. En esta Comunidad hay 2.723 hoteles abiertos (IECA, noviembre, 2016) de los cuales, 45 certificados con Q de calidad, lo que supone una implantación del 1,65\%. Asimismo, 127 hoteles distinguidos con SICTED (Calidad Turística, 2016), es decir, el 4,66\% de los establecimientos abiertos. La recopilación de los datos para el estudio, ha permitido encuestar a 169 páginas webs, puesto que 3 de los hoteles distinguidos por SICTED no disponen de página web $(1,7 \%)$. Se han realizado 1.480 preguntas para hoteles con la $\mathrm{Q}$ de calidad y 4.092 para hoteles SICTED.

\section{ANÁLISIS Y RESULTADOS}

\section{Resultados H1. ¿Están posicionados y son visibles dichos logotipos en las páginas webs y redes sociales?}

La visualización en las webs y redes sociales de los logotipos de los hoteles certificados es de gran importancia. Su posición en internet se entiende como el lugar de ubicación donde aparece el objeto de búsqueda. El resultado de la primera hipótesis informa que de los 172 hoteles que conforman la muestra (tabla 1), el 53\% no exhiben ningún logotipo de calidad turística. Es interesante destacar que el $63 \%$ de los establecimientos distinguidos con SICTED si lo presentan en las páginas principales frente al $27 \%$ de los certificados con Q de calidad. Es decir, tan sólo el $46 \%$ del total exponen sus logotipos visualmente a sus clientes de su acreditación turística.

El 5\% de los hoteles posiciona sus logotipos de acreditación Q y SICTED en la situación óptima de visualización (above the fold) para el usuario de la web. Y sólo el $32 \%$ de los hoteles acreditados exhiben su logotipo conforme a las medidas y colores que estipula el manual de identidad corporativa, con el tamaño adecuado para que pueda ser visualizado correctamente. 
En las redes sociales Facebook y Twiter, ninguno de los establecimientos hoteleros de la muestra exhibe en sus perfiles sociales los logotipos de acreditación turística. Por lo que la visualización es nula. Ante los presentes resultados se hace patente la baja visualización que tienen los logotipos de acreditación turística Q y SICTED en la web, llegando a ser inexistente en las redes sociales.

\section{Tabla 1}

\section{VISIBILIDAD DE LOS LOGOTIPOS}

\begin{tabular}{|c|c|c|c|c|c|c|}
\hline \multirow[b]{2}{*}{ Exhiben sus logotipos en la web } & \multicolumn{2}{|c|}{ Q Calidad } & \multicolumn{2}{|c|}{ SICTED } & \multicolumn{2}{|c|}{ Muestra } \\
\hline & Hoteles & $\%$ & Hoteles & $\%$ & Hoteles & $\%$ \\
\hline Hoteles con logotipo en home page & 33 & $73 \%$ & 44 & $35 \%$ & 77 & $46 \%$ \\
\hline Hoteles sin logotipo en home page & 12 & $27 \%$ & 80 & $65 \%$ & 92 & $54 \%$ \\
\hline \multicolumn{7}{|l|}{ Posicionamiento en la home page } \\
\hline Óptima visualización (Above the fold) & 6 & $13 \%$ & 3 & $2 \%$ & 9 & $5 \%$ \\
\hline Baja visualización (Below the fold) & 27 & $60 \%$ & 41 & $32 \%$ & 68 & $40 \%$ \\
\hline Visualización en páginas secundarias & 31 & $69 \%$ & 28 & $22 \%$ & 59 & $34 \%$ \\
\hline La imagen es correcta & 15 & $33 \%$ & 40 & $31 \%$ & 55 & $32 \%$ \\
\hline La imagen no es correcta & 19 & $42 \%$ & 34 & $27 \%$ & 53 & $31 \%$ \\
\hline \multicolumn{7}{|l|}{ Facebook } \\
\hline Exhiben logotipo en su perfil Facebook & 0 & $0 \%$ & 0 & $0 \%$ & 0 & $0 \%$ \\
\hline \multicolumn{7}{|l|}{ Twiter } \\
\hline Exhiben logotipo en su perfil Twiter & 0 & $0 \%$ & 0 & $0 \%$ & 0 & $0 \%$ \\
\hline
\end{tabular}

Fuente: Candau, 2016.

\section{Resultados H2: ¿Comunican estos hoteles a los clientes los beneficios que les ofrece el que sus servicios estén certificados?}

El 94\% de los establecimientos estudiados no comunican a sus clientes las ventajas que les suponen que sus servicios estén certificados en sus webs (tabla 2). En el caso de las redes sociales, este porcentaje se eleva al $99 \%$.

Sólo el $11 \%$ de los hoteles ofrecen a sus clientes explicaciones sobre las ventajas que supone la Q de calidad. El porcentaje disminuye al 4\% en los hoteles SICTED. Otras de las vías utilizadas por los establecimientos hoteleros para informar sobre sus certificados es la disposición de links a las webs de las entidades certificadoras. Esta vía es utilizada por el $9 \%$ de los mismos.

Los resultados de esta hipótesis revelan que la comunicación que se realiza de los logotipos de calidad Q y SICTED a los clientes es insuficiente para una correcta asimilación y entendimiento. La baja exposición visual, unida a la casi nula difusión de la significación de estos SGC turística tiene como consecuencia que el usuario de la web y redes sociales de los establecimientos, tenga un mínimo impacto en comparación del esfuerzo realizado por las empresas para vincular su marca a un certificado de calidad. Se debe resaltar que el $97 \%$ no exhiben ni sus políticas de calidad como tampoco sus diplomas acreditativos. 
Tabla 2

\section{COMUNICACIÓN A LOS CLIENTES}

\begin{tabular}{|c|c|c|c|c|c|c|}
\hline \multirow[b]{2}{*}{ Información sobre la acreditación } & \multicolumn{2}{|c|}{ Q Calidad } & \multicolumn{2}{|c|}{ SICTED } & \multicolumn{2}{|c|}{ Muestra } \\
\hline & Hoteles & $\%$ & Hoteles & $\%$ & Hoteles & $\%$ \\
\hline Explicación de la certificación en la web & 5 & $11 \%$ & 5 & $4 \%$ & 10 & $6 \%$ \\
\hline $\begin{array}{l}\text { No explicación de la certificación en } \\
\text { web }\end{array}$ & 40 & $89 \%$ & 119 & $96 \%$ & 159 & $94 \%$ \\
\hline $\begin{array}{l}\text { Hoteles con links a calidadturistica.org/ } \\
\text { calidadendestino.es }\end{array}$ & 4 & $9 \%$ & 12 & $9 \%$ & 16 & $9 \%$ \\
\hline $\begin{array}{l}\text { Hoteles sin links a calidadturistica.org/ } \\
\text { www.calidadendestino.es }\end{array}$ & 41 & $91 \%$ & 112 & $91 \%$ & 153 & $91 \%$ \\
\hline \multicolumn{7}{|l|}{ Diploma acreditativo } \\
\hline Hoteles que exhiben su acreditación & 1 & $2 \%$ & 4 & $3 \%$ & 5 & $3 \%$ \\
\hline \multicolumn{7}{|l|}{ Difusión } \\
\hline Publican la política de calidad & 5 & $11 \%$ & 1 & $1 \%$ & 6 & $3 \%$ \\
\hline \multicolumn{7}{|l|}{ Facebook } \\
\hline Informan de su certificado en Facebook & 3 & $7 \%$ & 0 & $0 \%$ & 3 & $2 \%$ \\
\hline Informan en otro apartado de Facebook & 1 & $2 \%$ & 1 & $1 \%$ & 2 & $1 \%$ \\
\hline \multicolumn{7}{|l|}{ Twiter } \\
\hline Informan de su certificado en Twiter & 2 & $4 \%$ & 0 & $0 \%$ & 2 & $1 \%$ \\
\hline
\end{tabular}

Fuente: Candau, 2016.

\section{Resultados H3: ¿Qué relevancia tienen los sellos influencers y sus premios de exce- lencia frente a los certificados de calidad?}

El metabuscador TripAdvisor tiene en su página web 1.775 hoteles andaluces referenciados (Tripadvisor, 2016) de los 2.723 establecimientos (IECA, 2016) lo que supone un $43 \%$, frente al 1,65\% de implantación de la certificación Q de Calidad y el 4,66\% que tiene la distinción SICTED. Este dato por si sólo confirma la gran aceptación que ha tenido tanto a nivel empresarial como de usuario el sello influencers TripAdvisor.

En la muestra de hoteles, la presencia de sellos influencers en las páginas principales de sus webs asciende al $24 \%$ y casi todos ellos, concretamente el $23 \%$ tienen un tamaño igual o superior a los logotipos de calidad Q y SICTED, siendo un 15\% de mayor tamaño, lo que demuestra la importancia que dan los empresarios a este sello, incluso por encima de los sistemas de calidad certificados por entidades dedicadas en exclusiva a la calidad como Q y SICTED.

El que tiene más presencia en las webs de los hoteles es Tripadvisor. Curiosamente este sello influencers se creó en el año 2010, diez años después que el SICTED y catorce años posteriores a la $\mathrm{Q}$ de calidad. Aún así, tiene una presencia del $23 \%$ en la página 
principal de las webs y con un tamaño mayor ó igual que los logotipos Q y SICTED. Si profundizamos se comprueba que el $11 \%$ de la muestra exhibe el premio de excelencia de la calidad y no el logotipo Q ni SICTED.

Tabla 3

RELEVANCIA DE LOS SELLOS INFLUENCERS

\begin{tabular}{|l|c|c|c|c|c|c|c|}
\hline & \multicolumn{2}{|c|}{ Q Calidad } & \multicolumn{2}{|c|}{ SICTED } & \multicolumn{2}{c|}{ Muestra } \\
\hline Presencia de los sellos influencers & Hoteles & $\%$ & Hoteles & $\%$ & Hoteles & $\%$ \\
\hline Hoteles que exhiben sellos influencers & 7 & $16 \%$ & 35 & $28 \%$ & 42 & $24 \%$ \\
\hline Presencia de: & & & & & & \\
\hline & 4 & $9 \%$ & 19 & $15 \%$ & 23 & $13 \%$ \\
\hline \multicolumn{1}{|l|}{ Tripadvisor } & 3 & $7 \%$ & 9 & $7 \%$ & 12 & $7 \%$ \\
\hline & - & - & 9 & $7 \%$ & 9 & $5 \%$ \\
\hline Srivago & 1 & $2 \%$ & 0 & $0 \%$ & 1 & $1 \%$ \\
\hline Sellos con óptima visualización & 6 & $13 \%$ & 20 & $16 \%$ & 26 & $15 \%$ \\
\hline Sellos influencers > que Q y SICTED & 0 & $0 \%$ & 14 & $11 \%$ & 14 & $8 \%$ \\
\hline Sellos influencers con links en sus páginas & 2 & $4 \%$ & 21 & $17 \%$ & 23 & $13 \%$ \\
\hline Premios de excelencia & & & & & & \\
\hline Exhiben premio de excelencia & 11 & $24 \%$ & 31 & $24 \%$ & 42 & $24 \%$ \\
\hline Premio a la excelencia de Tripadvisor & 11 & $24 \%$ & 29 & $23 \%$ & 40 & $23 \%$ \\
\hline Situados con óptima visualización & 3 & $7 \%$ & 2 & $2 \%$ & 5 & $3 \%$ \\
\hline Premios de excelencia > que Q y SICTED & 7 & $16 \%$ & 20 & $16 \%$ & 27 & $16 \%$ \\
\hline Premios de excelencia = que Q y SICTED & 4 & $9 \%$ & 10 & $8 \%$ & 14 & $8 \%$ \\
\hline Exhiben el premio, no logotipo QSICTED & 3 & $7 \%$ & 16 & $37 \%$ & 19 & $11 \%$ \\
\hline Premios de excelencia linkados a web & 5 & $11 \%$ & 20 & $16 \%$ & 25 & $15 \%$ \\
\hline
\end{tabular}

Fuente: Candau, 2016.

En cuanto a la comunicación que realizan los premios de excelencia y calidad, el $15 \%$ dispone de link informativo sobre lo que supone tener este reconocimiento. Hay que recordar que los establecimientos que linkan a la página de calidad turística/calidad en destino asciende al $9 \%$. Por este motivo, la comunicación con su público es más fluida.

Tras estos resultados se puede confirmar que los sellos influencers otorgados por las agencias on-line están mejor valorados por los empresarios hoteleros que los certificados de calidad Q y SICTED. Las razones que lo explican son su introducción en el mercado, su posicionamiento y ponderación visual en las webs. Esto permite que estén mejor asimilados por el usuario que interactúa con los mismos. 


\section{Resultados H4: ¿Han amoldado los hoteles su gestión de calidad e interacción con los clientes a la era 2?0?}

Las redes sociales facilitan a los hoteles conocer más sobre sus clientes y adaptar sus mensajes a las nuevas demandas de consumidores, ayudando a crear relaciones estrechas (Harrigan et al., 2017). El 26\% de establecimientos están al margen de las redes sociales, pero la mayoría el $72 \%$ tienen perfil en las mismas. Facebook es la red social donde hay mayor presencia con un $100 \%$ de los establecimientos. El uso que hacen los establecimientos de sus perfiles es de imagen de marca. En ninguno de los casos es utilizada como vía de atención y servicio al cliente, eliminando el factor de interactividad en este punto.

Cabe destacar que el $15 \%$ del total de la muestra tiene habilitada una línea de atención al cliente bidireccional en sus páginas webs. La comunicación bidireccional a través de la web con el cliente es una forma de hacerle más fácil la reserva ó la resolución de preguntas que le pueda surgir. Ésta está al alcance de aquellos establecimientos que dispongan de página web y de una organización adecuada a la atención de clientes. Cabe destacar que es una tendencia que irá aumentando y que los primeros en ponerla a disposición fueron algunas grandes cadenas hoteleras, tales como Paradores.

Tabla 4

INTERACCIÓN CON EL USUARIO

\begin{tabular}{|l|c|c|c|c|c|c|c|}
\hline & \multicolumn{2}{|c|}{ Q Calidad } & \multicolumn{2}{c|}{ SICTED } & \multicolumn{2}{c|}{ Muestra } \\
\hline Interacción con el cliente en las webs & Hoteles & $\%$ & Hoteles & $\%$ & Hoteles & $\%$ \\
\hline Hoteles con línea bidireccional & 17 & $38 \%$ & 9 & $7 \%$ & 26 & $15 \%$ \\
\hline Libro de quejas y/o sugerencias on-line & 0 & $0 \%$ & 0 & $0 \%$ & 0 & $0 \%$ \\
\hline Comentarios con los clientes & 23 & $51 \%$ & 34 & $27 \%$ & 57 & $33 \%$ \\
\hline Hoteles con links a redes sociales & 41 & $91 \%$ & 83 & $65 \%$ & 124 & $72 \%$ \\
\hline Hoteles con links a Facebook & 41 & $91 \%$ & 83 & $65 \%$ & 124 & $72 \%$ \\
\hline Hoteles con links a Twiter & 37 & $82 \%$ & 72 & $57 \%$ & 109 & $63 \%$ \\
\hline Hoteles no conectados a redes sociales & 4 & $9 \%$ & 41 & $32 \%$ & 45 & $26 \%$ \\
\hline $\begin{array}{l}\text { Hoteles que usan las redes sociales } \\
\text { como atención al cliente }\end{array}$ & 0 & $0 \%$ & 0 & $0 \%$ & 0 & $0 \%$ \\
\hline
\end{tabular}

Fuente: Candau, 2016.

Ninguno de los establecimientos dispone de un libro de sugerencias, agradecimientos y quejas on-line, aunque destaca que el $33 \%$ de ellos gestiona los comentarios de clientes en su misma página, con lo que se presupone interactividad con el usuario, además de la gestión de venta.

Aunque el uso de las TICs está introducido en las empresas hoteleras, éstas no van más allá de la exposición de su imagen. Por esta razón, se entiende la necesidad de implementación de herramientas 2.0 que posibiliten y fomenten la interacción, no sólo con sus clientes sino con todos sus stakeholders, con objeto de poder diseñar estrategias que la 
posicionen estratégicamente respecto a su competencia, ya sea esta diferenciación a través de la atención al cliente, colmando sus expectativas ó produciendo un servicio diferente a lo existente en el mercado.

\section{CONCLUSIONES}

El rápido desarrollo de las TICs y la implementación de la tecnología 2.0, ha cambiado drásticamente la industria del turismo (Ho y Lee, 2007). Está ampliamente aceptado que internet es una herramienta de marketing eficaz en el sector turístico, tanto para proveedores como consumidores, de gran valor en la difusión de información, comunicación, ventas y retroalimentación. Las empresas turísticas, orientadas a clientes, están adoptando los modelos que implantan la tecnología 2.0 para alcanzar sus objetivos, al ser una de las más influyentes en el cambio de comportamiento de los turistas (Buhalis y Law, 2008). Por ende, toda organización, sistema ó empresa que esté dentro del sector turístico debe adaptarse a la era digital y a las consiguientes evoluciones que se produzcan, los que no se amolden quedarán excluidos.

Las conclusiones del trabajo empírico demuestran de que los actuales SGC turística, Q y SICTED, han obviado de sus sistemas los factores que generan más valor en los nuevos consumidores como son: el posicionamiento, la visibilidad, la comunicación y la interactuación.

La Falta de posicionamiento y casi nula visualización optima, que tienen a día de hoy los sistemas de gestión de calidad turística, Q y SICTED, (Sólo el 5\% de los logotipos del total los establecimientos están óptimamente posicionados en sus webs. Siendo el $0 \%$ visibilidad y posicionamiento en redes sociales). Unida a la deficiente comunicación informativa que ofrecen a sus usuarios (El 94\% de los establecimientos no comunican a sus clientes el significado de la certificación Q y SICTED en sus webs. Y sólo un $2 \%$ lo comunican en redes sociales) y coronada con la baja interacción mantenida con los usuarios (del total de la muestra, un 15\% dispone de línea bidireccional de comunicación con los clientes, estando establecidas todas ellas en web. No se utiliza ninguna red social para este propósito sino como imagen de marca. Cabe destacar que el $33 \%$ gestiona los comentarios que sus clientes realizan en sus páginas webs), concluye la falta de adecuación de los actuales SGC turísticos a la cuarta revolución industrial.

La falta de implementación de las actuales estrategias de marketing asociadas a la tecnología 2.0, para potenciar sus marcas como referente de confianza han provocado el desinterés por parte del empresariado, razón a la que se debe el estancamiento en el crecimiento de nuevas implantaciones de estos SGC en hoteles. Que desde hace más de diez años se mantienen en las mismas cuotas de mercado (Camisón, 2004).

Paradójicamente sorprende el crecimiento de los sellos influencers y premios de excelencia otorgados por los metabuscadores, que ha despertado el interés de la red empresarial al conquistar a los usuarios. El crecimiento exponencial que han tenido los metabuscadores demuestra que el público está interesado en la calidad de los establecimientos. Los Sellos Influencers han sabido posicionar a los nuevos consumidores en el centro de sus sistemas convirtiéndolos en colaboradores imprescindibles de los mismos, mediante una continua interacción que genera la comunicación, la retroalimentación y la 
transparencia de los procesos. Mejorando la visibilidad y a venta de los servicios. Han desplazado del mercado a los SGC turística como referentes de confianza a la hora de su toma de decisión en la compra de servicios turísticos.

Hoy día, los usuarios depositan su confianza en los sellos influencers, que obvian la certificación y auditorías de las estructuras y gestión interna de los establecimientos, basándose únicamente en opiniones y referencias de otros clientes. Esta debilidad es la oportunidad que tienen los SGC Turística para lograr reconquistar la confianza de los clientes y empresarios. La etapa en que se consideraba a los SGC como una ventaja competitiva en el sector del turismo (Aaker 2010; Ollé y Riu, 2009) ha dejado de existir. Recuperar la confianza de clientes y empresarios, es el objetivo que deben marcarse los nuevos SGC turística. De lo contrario, la actual tendencia decreciente de implantación, seguirá de forma continua hasta la desaparición de los mismos.

Los nuevos tiempos exige a los SGC turística que transiten de ser herramientas de una correcta gestión empresarial, a ser garantes y abanderados de una mejora continua que sea visible, comunicada, retroalimentada y vendida a los nuevos consumidores. Para conseguir este objetivo deberán implementar las tecnología 2.0, posicionando en el centro de sus sistemas a los clientes, incluyendo en sus procesos los factores del marketing que generan más valor en los nuevos para ellos, como son: el posicionamiento, la visibilidad, la comunicación y la interactuación. Deberán poner en valor la seguridad que ofrece un establecimiento certificado por una entidad acreditada, frente a aquellos que disponen de un sello influencers que solamente atiende a la prescripción de los clientes, para fundamentar la calidad. Si se pretende que la calidad ofrecida sea la esperada, la entrada de la etapa de la calidad colaborativa es inevitable.

Siguiendo esta filosofía la línea aérea Vueling utiliza su cuenta "Vueling Airlines" de Twiter como canal oficial de servicio a clientes, en la misma informa y atiende las quejas de sus clientes, con un tiempo máximo de respuesta de 15 minutos. Las líneas aéreas como el caso de Vueling o Ryanair, también disponen de una línea de atención al cliente, y son las primeras que están ajustando sus sistemas de calidad a las TICs ganando en satisfacción, inmediatez y proximidad a sus usuarios.

Esta imposición que demanda el mercado es un revulsivo que se hace extensible no sólo a los SGC turística sino a todos los SGC que se están obligados a ser revisados, y amoldarse en cada uno de sus sectores a la Calidad Colaborativa. En esta era, internet y las TIC's se ha posicionado como vía imprescindible para los individuos, poniendo en el punto estratégico a los clientes si se desea atraerlos y fidelizarlos. Si los SGC desean conocer la satisfacción del cliente, ¿qué mejor manera que facilitarle el camino digital? La calidad turística debe ajustar sus SGC a la calidad colaborativa:

- Adaptando sus procedimientos la interactividad con los usuarios finales y demás stakeholders, generando transparencia en sus procedimientos.

- Promoviendo la formación de una red de colaboración y creación de conocimientos que propicie la mejora continua de los servicios.

- Estableciendo un sistema coordinado bajo los criterios éticos establecidos por la empresa, que alcance la satisfacción del cliente de forma funcional, emocional y espiritual. 
- Convirtiéndose en herramientas ágiles que fomenten la inmediatez y la cercanía en la resolución de incidencias, al tiempo que aseguren la confianza y fidelización de clientes, proveedores y empleados.

\section{BIBLIOGRAFÍA}

AAKER, D. (2010): «Marketing challenges in the next decade». Journal of Brand Management 17, pp. 315-316.

ÁLVAREZ, J., DEL RÍO, M.C., DURÁN, A. y URBANO, B. (2017): «Sistema de calidad turístico español: revisión de la literatura». Ágora, 19 (2), 4-13.

BORRÁS, J., MORENO, A. y VALLS, A. (2014): «Intelligent tourism recommender systems: A survey». Expert Systems with Applications, 41 (16), 7.370-7.389.

BRODIE, R.J., HOLLEBEEK, L.D., JURIC, B. e ILIC, A. (2011): «Customer engagement: Conceptual domain, fundamental propositions, and implications for research». Journal of service research, 14 (3), 252-271.

BUHALIS, D. y LAW, R. (2008): «Progress in information technology and tourism management: 20 years on and 10 years after the Internet- The state of eTourism research». Tourism Management, 29 (4), 609-623.

CALIDAD TURISTICA (2016): Información General SCTE (Sistema de Calidad Turística Española).25 de noviembre 2016. Disponible en http://www.profesionales.calidadturistica.es/index.aspx

CAMISÓN, C. (2004): «Estrategias de Calidad Turística. El papel de los sistemas de acreditación y certificados de calidad». Mediterráneo Económico. Nueva Formas el Turismo 5. Almería, Cajamar, 105-169.

CANDAU, M. (2016): La calidad turística en la era 2.0: Estudio del SICTED y la Q en las webs de los hoteles certificados en Andalucía. Trabajo de Fin de Máster. Universidad de Sevilla.

CANTALLOPS, A.S. y SALVI, F. (2014): «New consumer behavior: A review of research on eWOM and hotels». International Journal of Hospitality Management, 36, 41-51.

CARRASCO, G. y LORENZO, S. (2015): «Las redes de innovación colaborativa y el futuro de la calidad asistencial». Revista de Calidad Asistencial, 30 (2), 53-54.

CASANUEVA, C., CASTRO, I. y GALÁN, J.L. (2013): «Informational networks and innovation in mature industrial clusters». Journal of Business Research, 66 (5), 603613.

CASTELLÓ MARTÍNEZ, A. y DEL PINO, C. (2015): «La comunicación publicitaria con influencers». Redmarka: Revista académica de marketing aplicado, 14, pp. 21-50.

CELAYA, J. (2008): La empresa en la Web 2.0. Madrid, Gestión 2000.

CENTINKAYA, A. S. (2010). «Information and Communication Technologies in Tourism 2008». Tourism Management. In Proceedings of the International Conference in Innsbruck. 591.

CHAVES, L. (2006): «Comunicación publicitaria eficaz: del concepto a la medida». Harvard Deusto Marketing y Ventas, 77, 64-71.

CODINA, L. y MARCOS, M.C. (2005): «Posicionamiento web: conceptos y herramientas». El profesional de la información, 14 (2), 84-99. 
COSTA, J. (2014): El DirCom hoy: Dirección y Gestión de la Comunicación en la nueva economía. Barcelona, Ediciones Costa Punto Com.

CRAVERO IGARZA, S. y ALZA, M.E. (2012): «Comunicación de destinos en tiempos de social media marketing». Notas en Turismo y Economía. Año III (V), 7-27.

CRICK, A.P. y SPENCER, A. (2011). «Hospitality quality: new directions and new challenges». International Journal of Contemporary Hospitality Management, 23 (4), 463-78.

CUESTA, F. y ALONSO, M.A. (2010): Marketing directo 2.0. Cómo vender más en un entorno digital. Barcelona, Gestión 2000.

DE BRUIN, R., KEULEERS, E., LAZARO, C., POULLET, Y. y VIERSMA, M (2005): Analysis and definition of common characteristics of trustmarks and web seals in the European Union.

DEL PINO, C., MARTÍNEZ, A. C., y SOLER, I. R. (2013): La comunicación en cambio constante: Branded Content, Community Management, Comunicación 2.0. Madrid, Editorial Fragua.

DEZA, M. (2007): Consumidores nómadas. El siglo del mobile marketing. La Coruña, Netbiblo.

DIANA-JENS, P. y RUIBAL, A.R. (2015): «La reputación online y su impacto en la política de precios de los hoteles». Cuadernos de Turismo, 36, 129-155.

DOMINGO, G. (2013): «Innovación de marca: la apuesta digital». En Zilles, Cuenca, J. y Rom, J. (Eds.) Breaking the Media Value Chain VII International Conference on Communication and Reality, 101-107.

ERRAGCHA, N. y ROMDHANE, R. (2014): «New faces of marketing in the era of the web: from marketing 1.0 to marketing 3.0». Journal of Research in Marketing, 2 (2), 137-142.

ESTEBAN TALAYA, Á. (2014): «Los nuevos desarrollos turísticos en España y su efecto en la recuperación y/o promoción económica». Papers de Turisme, 17, 33-44.

FERNÁNDEZ RODRÍGUEZ, C. (2013). «El valor de lo intangible y armonizado en la calidad turística europea». Revista de Derecho de la Unión Europea, 24, 343-372.

FERNÁNDEZ-CAVIA, J. y HUERTAS-ROIG, A. (2010): "City brands and their communication through web sites: Identification of problems and proposals for improvement". En Web Technologies: Concepts, Methodologies, Tools, and Applications. Hershey, Information Science Reference, IGI Global, 1.174-1.297.

FERNÁNDEZ-CAVIA, J., VINYALS-MIRABENT, S. y LÓPEZ-PÉREZ, M. (2013): Calidad de los sitios web turísticos oficiales de las comunidades autónomas españolas. BiD: Textos universitaris de biblioteconomía i documentació, $\mathrm{n}^{\circ} 31$.

GARCÍA HENCHE, B. (2005): «Características diferenciales del producto turismo rural». Cuadernos de Turismo, 15, 113-134.

GODIN, S. (2008): La Vaca Púrpura. Barcelona, Ediciones Gestión 2000.

GONZÁLEZ, L., GONZÁLEZ, C., JUANEDA, E. y PELEGRIN, J. (2014): La calidad en las organizaciones turísticas. Madrid, Ediciones Paraninfo.

GONZALEZ, Z. y CONTRERAS, R. (2012): «Apps como una posibilidad más de comunicación entre la marca y su público: un análisis basado en la valoración de los usuarios». Pensar la Publicidad, 6 (1), 81-100. 
HARRIGAN, P., EVERS, U., MILES, M., y DALY, T. (2017): «Customer engagement with tourism social media brands». Tourism Management, 59, pp. 597-609.

HARRIS, L. y RAE, A. (2009): «Social networks: the future of marketing for small business». Journal of Business Strategy, 30 (5), 24-31.

HENNING-THURAU, T., MALTHOUSE, E. C., FRIEGE, C., GENSLER, S., LOBSCHAT, L., RANGASWAMY, A. y SKIERA, B. (2010): «The impact of new media on customer relationships». Journal of service research, 13 (3), 311-330.

HERNÁNDEZ-MÉNDEZ, J., MUÑOZ-LEIVA, F., y SÁNCHEZ-FERNÁNCHEZ-FERNÁNDEZ, J. (2015). «The influence of e-word-of-mouth on travel decision-making: consumer profiles». Current Issues in Tourism, 18 (11), 1.001-1.021.

HO, C.I. y LEE, Y.L. (2007): «The development of an e-travel service quality scale». Tourism Management, 28 (6),1.434-1.449.

HUERTAS-GARCÍA, R. (2005): «Eficiencia de la implantación de sistemas de calidad. Análisis de las empresas hoteleras». Estudios Turísticos, 165, 33-55.

IECA (Instituto de Estadistica y Cartografía de Andalucía) (2016): Personal empleado, establecimientos abiertos estimados, plazas estimadas y grados de ocupación. Sevilla, Junta de Andalucía. 25 de octubre de 2016. Disponible en:http://www.juntadeandalucia.es/institutodeestadisticaycartografia/iea

INGLADA, M.E. y SASTRE, J.M. (2014): «Reflexiones sobre la globalización, pobreza y desarrollo: incidencia en las organizaciones de economía social». REVESCO: Revista de estudios cooperativos, 116, 160-179.

KAPLAN A.M. y HAENLEIN M, (2010): «Users of the world, unite! The challenges and opportunities of social media», Business Horizons, 53 (1), 59-58.

KIRÁL'OVÁ, A. y PAVLÍČEKA, A. (2015). «Development of social media strategies in tourism destination». Procedia-Social and Behavioral Sciences, 175, 358-366.

KOTLER, P., KARTAJAYA, H., y SETIAWAN, I. (2011): Marketing 3.0. Madrid, LID Editorial Empresarial.

LAI, I. K.W. (2014). «The role of service quality, perceived value, and relationship quality in enhancing customer loyalty in the travel agency sector». Journal of Travel \& Tourism Marketing, 31 (3), 417-442.

LAJA, P. (2013): How to Build Websites that Sell: The Scientific Approach to Websites. Hyperink Ebooks.

LAW, R., QI, S. y BUHALIS, D. (2010): «Progress in tourism management: A review of website evaluation in tourism research». Tourism Management, 31 (3), 297-313.

LÓPEZ JIMÉNEZ, D., REDCHUK, A., DITTMAR, E.C. y VARGAS, J.P. (2013): «Los logotipos de privacidad en Internet: percepción del usuario en España». RISTI-Revista Ibérica de Sistemas e Tecnologias de Informação, 12, 49-63.

LÓPEZ JIMÉNEZ, D. y REDCHUK, A. (2015): «Tendencias sociales en el comercio electrónico de América Latina: a propósito de los proveedores adheridos a códigos de buenas prácticas». Opción, 31 (78), 67-79.

LÓPEZ JIMÉNEZ, D. y CASTILLO GIRÓN, V.M. (2016): «Prácticas empresariales de excelencia en Internet: la exhibición de logotipos de garantía en los sitios web». Mercados y Negocios, 23-24, 71-88. 
LUNA-NEVAREZ, C. e HYMAN, M. (2012): «Common practices in destination website design». Journal of Destination Marketing \& Management, 1 (12), 94-106.

MELLINAS, J.P., MARTINEZ, S.M. y BERNAL, J.J. (2016): «El uso de redes sociales por los hoteles como indicativo de gestión eficiente». Tourism \& Management Studies, 12 (2), 78-83.

MURPHY, J., SCHEGG, R. y OLARU, D. (2006): «Investigating the evolution of hotel internet adoption». Information Technology \& Tourism, 8 (3-1), 161-177.

NOVO VÁZQUEZ, A. (2014): «Consumocracia. El consumo político como forma de participación de la ciudadanía». Política y Sociedad, 51 (1), pp. 121-146.

OLLÉ, R. y RIU, D. (2009): El nuevo Brand Management. Cómo plantar marcas para hacer crecer negocios. Barcelona, Ediciones Gestión 2000.

PALAFOX, J. (Coord) (2014): Los tiempos cambian. Historia de la Economía. Valencia, Tirant Humanidades.

PARRA LÓPEZ, E. y MARTINEZ GONZÁLEZ, J.A. (2014): «Social media como estrategia de e-marketing: el caso de facebook en los hoteles de Canarias». En Congreso AECIT Turismo: liderazgo, innovación y emprendimiento, 1-10.

RICELlY PINTO DE FARIAS, T., COSTA, J.H., PERINOTTO, C., y RIANI, A. (2017): «Empresas turísticas, redes sociales y negocios electrónicos: Reflexiones a partir de la obra de Manuel Castells». Estudios y Perspectivas en Turismo, 26 (3), 626-643.

SANAGUSTÍN, E. (2016). Vender más con marketing digital. Bogotá, Ecoe Ediciones.

SCHEGG, R., LIEBRICH, A, SCAGLIONE, M. y AHMAD, S.F.S. (2008): «An exploratory field study of Web 2.0 in Tourism». En Information and Communication Technologies in Tourism. Viena, Springer

SCHWAB, K. (2016): La cuarta revolución industrial. Barcelona, Editorial Debate.

SCHWARTZ, D.G. (2009) «Toward the next generation of social networking applications». Internet Research, 19 (3). https://doi.org/10.1108/intr.2009.17219caa.001

SIMMONS, G.J. (2007): «i-Branding: developing the internet as a branding tool», Marketing Intelligence \& Planning 25 (6), 544-562.

SUBRAMI, M.R. y RAJAGOPALAN, B. (2003): «Knowledge-Sharing and Influence in Online Social Networks via Viral Marketing». Communications Of The ACM, 46 (12), 300-307.

TRIPADVISOR (2016): Establecimientos hoteleros en Andalucía. 27/11/2016. Disponible en https://www.tripadvisor.es/Hotels-g187428-Andalucia-Hotels.html

VAN DOORN, J., LEMON, K. N., MITTAL, V., NASS, S., PICK, D., PIRNER, P. y VERHOEF, P. C. (2010): «Customer engagement behavior: Theoretical foundations and research directions». Journal of Service Research, 13 (3), 253-266.

VELILLA, J. (2010): Branding. Tendencias retos en la comunicación de marca. Barcelona, Universitat Oberta de Catalunya.

VELEDO, R. (2016): Redes sociales como instrumento de comunicación de hoteles. Estudio sobre el uso de redes sociales por los clientes de hoteles. Trabajo Fin de Grado. Universidad de León.

VIEITES, Á.G. y BARROS, C.O. (2011): Redes sociales en la empresa: la revolución e impacto a nivel empresarial y profesional. Paracuellos de Jarama, Editorial Ra-Ma. 


\section{Anexo I}

COMPARATIVA DE LA EVOLUCIÓN DEL MARKETING Y LA CALIDAD

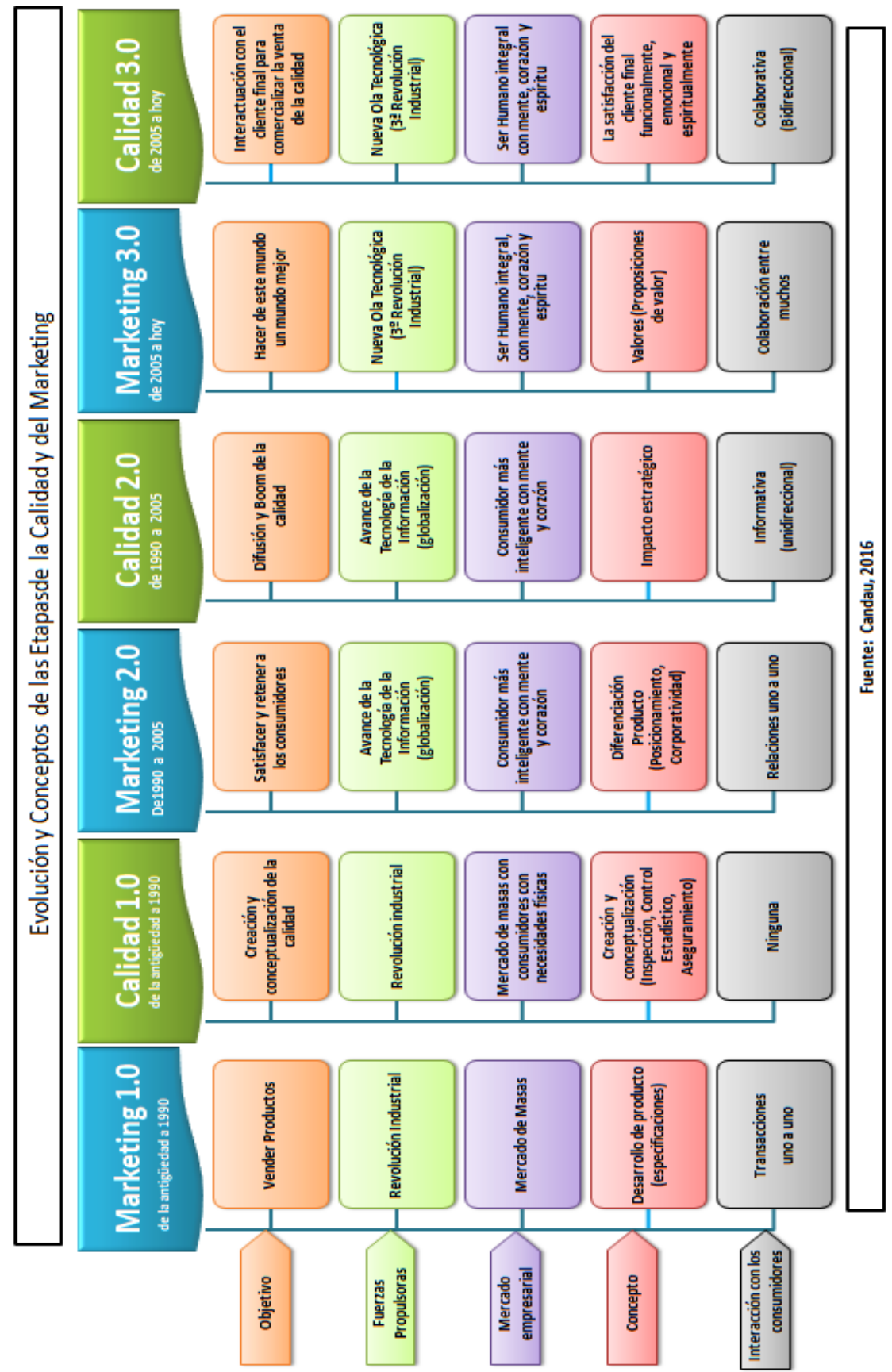




\section{Anexo II}

EVOLUCIÓN Y CONCEPTOS DE LAS ETAPAS DE LA CALIDAD A LA CALIDAD COLABORATIVA

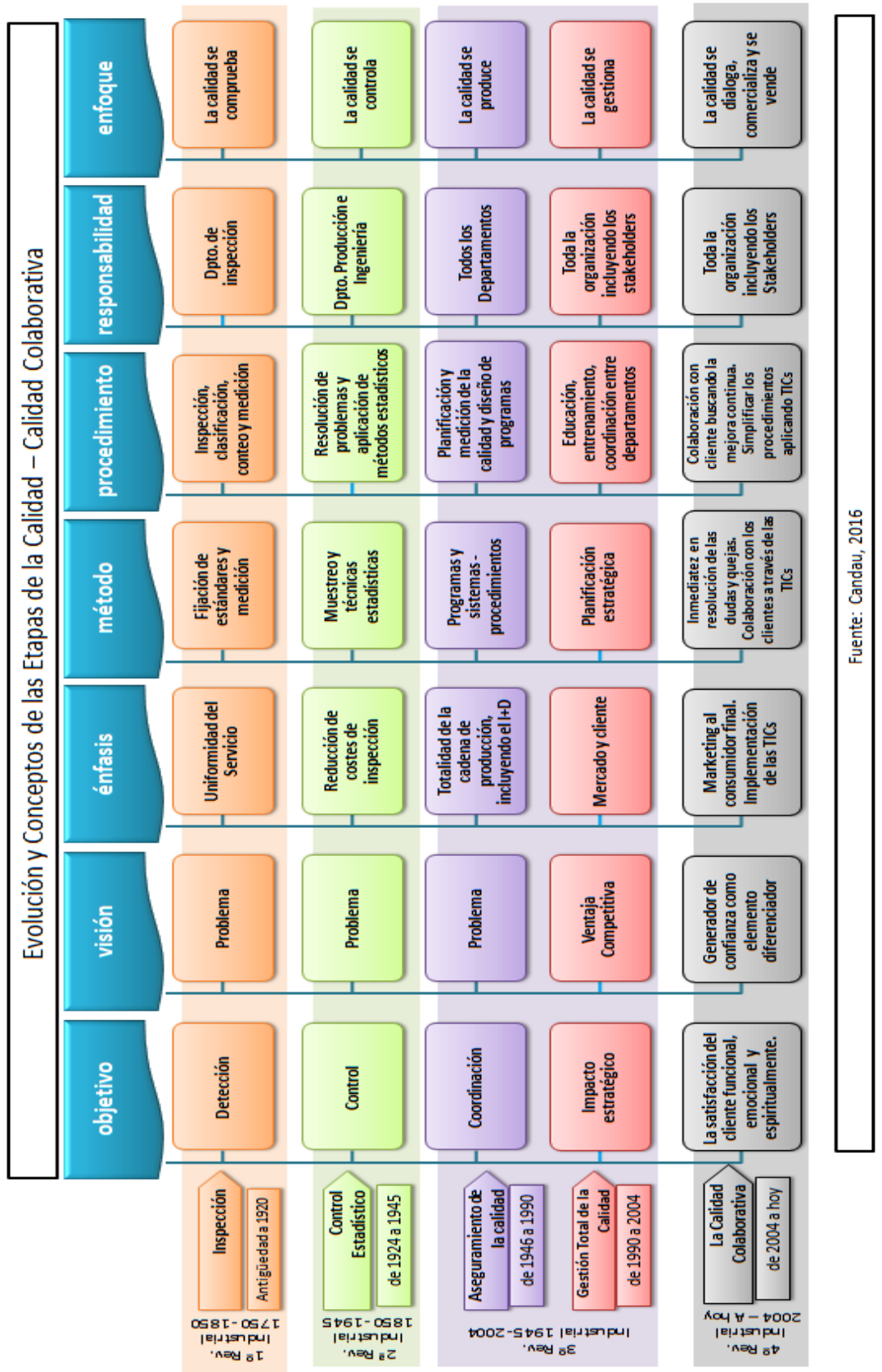




\section{Anexo III \\ CUESTIONARIO DEL TRABAJO EMPÍRICO A LOS ESTABLECIMIENTOS HOTELEROS DE ANDALUCÍA CON Q DE CALIDAD/SICTED}

\begin{tabular}{|c|c|}
\hline 1 & El logotipo acreditativo aparece en la home page \\
\hline 2 & El logotipo está situado "above the fold" \\
\hline 3 & El logotipo aparece en algún otro apartado \\
\hline 4 & La imagen del logotipo es correcta \\
\hline 5 & La web dispone de un link a la página de calidad turística/calidad en destino \\
\hline 6 & En la web, existe un espacio explicativo de la Q /SICTED \\
\hline 7 & Los clientes pueden visualizar el diploma acreditativo \\
\hline 8 & Los clientes pueden visualizar si está en vigor el certificado \\
\hline 9 & En la web está colgada la política de calidad \\
\hline 10 & En la home page se exhiben sellos influencers \\
\hline 11 & Qué tipo de sellos influencers se exhiben \\
\hline 12 & El sello infuencers está situado "above the fold" \\
\hline 13 & Los logotipos SICTED/Q son de mayor tamaño que los logotipos de guías turísticas \\
\hline 14 & Los logotipos SICTED/Q son de igual tamaño que los logotipos de guías turísticas \\
\hline 15 & Los sellos influencers linkados a páginas que amplifican y comunican lo que son \\
\hline 16 & $\begin{array}{l}\text { Aparecen premios de calidad otorgados por los clientes a través de agencias de viaje on- } \\
\text { line (Tripadvisor, Trivago, Kayak, etc.)? }\end{array}$ \\
\hline 17 & Qué tipo de reconocimientos de calidad se exhiben \\
\hline 18 & Los premios de excelencia están "above the fold" \\
\hline 19 & Los reconocimientos de calidad son de mayor tamaño que logotipo Q/ SICTED \\
\hline 20 & Los reconocimientos de calidad son de igual tamaño que logotipo Q/SICTED \\
\hline 21 & Los reconocimientos de calidad son links a sus páginas principales \\
\hline 22 & En la web existe un espacio para la atención online del cliente \\
\hline 23 & En la web, hay libro de quejas on-line \\
\hline 24 & En la web se pueden incluir los comentarios de clientes \\
\hline 25 & La web del hotel tiene links a Twiter \\
\hline 26 & La web del hotel tiene links a Facebook \\
\hline 27 & El perfil de Facebook exhibe el logotipo Q / SICTED \\
\hline 28 & En Facebook se encuentra el logotipo Q/SICTED \\
\hline 29 & En Facebook se informa del sistema Q/SICTED \\
\hline 30 & La web tiene links a Twiter \\
\hline 31 & El perfil de twiter exhibe el logotipo Q/SICTED \\
\hline 32 & La cuenta de twiter informa acerca del sistema Q /SICTED \\
\hline 33 & Las redes sociales, con links en la web, están destinadas a la atención al cliente online \\
\hline
\end{tabular}

Fuente: Candau, 2016. 